\title{
Study on PUF based Secure Protection for IC Design
}

\author{
${ }^{1}$ Wei Liang, ${ }^{1}$ Bo Liao, ${ }^{1}$ Jing Long, ${ }^{1}$ Yan Jiang, ${ }^{1,2}$ Li Peng \\ ${ }^{1}$ College of Mathematics and Econometrics, Hunan University changsha, Hunan, 410082, \\ China idlink@163.com,\{liaobo,jlong, yjiang $\} @$ hnu.edu.cn \\ ${ }^{2}$ School of Computer Science and Engineering, Hunan University of Science and Technology, \\ Xiangtan, 411201, China \\ lpeng@hnu.edu.cn
}

\begin{abstract}
The rapid progress in integrated circuit (IC) technology makes the gates in a single chip increase by Moore's law. The complexity in design and verification grows accordingly. To address this issue, intellectual property (IP) reuse is prevalently used in system design. However, it may incur IP piracy or illegal copy, which brings big threat to IP protection. When IP dispute occurs, the ownership verifier can activate the Challenge Response Pair (CRP) of Physical Unclonable Function (PUF) to verify IP copyright. In this work, we have analyzed and summarized PUF techniques and current research status. Furthermore, several typical PUF techniques are concretely illustrated and compared. Besides, we demonstrate PUF in multiprocessor by analyzing security and overhead on previous PUF techniques. Finally, we envision the future of PUF techniques and their applications.
\end{abstract}

Keywords. IC; IP; reuse; PUF; CRP

\section{Introduction}

In recent years, electronic products are widely used in people's daily life and the security issue attracts more and more attention [1]. There are many challenges to security, such as counterfeiting, cloning, reversing engineering and vicious addition of components. Although popular reuse technology has brought great convenience to Semiconductor Company, it makes Integrated Circuit (IC) copyright easy to be 
misused. The semiconductor companies are suffering tremendous financial loss, e.g., cable television. Illegal user may copy the circuit in the set top box and crack the secret key. They will enjoy free television service through cracking [2]. Popularly, identification system realizes ownership protection by inserting a Radio Frequency Identification (RFID) label into one of the Intellectual Property (IP) cores [3-6]. However, the insertion lacks of secure protection, illegal attackers can still read the sensitive information in RFID label and clone a new card with the same functionality surreptitiously. It causes damage to benefits of Cable Television Company. Consequently, it is crucial to protect IP components in IC design by utilizing secure reliable mechanisms.

Generally, immature protection techniques bring big challenges to forensics and judicial authentication. The confidence crisis in IP ownership presses for effective protection techniques in order to deter IP infringement. Recently, researchers have proposed many IP watermarking methods [7-11]. These methods embed watermarks at various abstract levels, but cause growth of hardware resource, such as area, speed and power consumption. Furthermore, the performance of IP circuit is also affected. Therefore, there are many bottleneck issues to be solved in IP protection methods.

With the rapid advance of IC, Physical Unclonable Function (PUF) [12-15] emerges as a new technique to identify a single physical device. It is a chip-dependent unclonable challenge-response function that can uniquely identify a specific integrated circuit. The physical features of device are utilized to realize the mapping from an input signal to an unpredictable output.

The unique structure of PUF utilizes many random factors in manufacturing. So, it is hard to be forged. We utilize the notations $C, R, F$ to denote the challenge of PUF, response of PUF and the mapping relationship respectively. There are four characteristics of PUF techniques, summarized as follows.

(1)Unpredictable. Given a specific PUF, it is hard to derive $F$ from a set of challenge-responses $Q=\left\{C_{i}, R_{i}\right\}, i=0,1,2, \ldots q$. To add a new challenge $C_{q+i}$ to this structure, it is almost impossible to correctly predict $R_{q+i}$.

(2)Non-repeatability. Even with precise manufacturing technology and the same CRP, nobody can produce a same PUF structure $B$ with the existing PUF $A$, both of which have the same mapping relationship. 
(3)Uniqueness. The entities $A$ and $B$ produced by the same PUF have various mapping relationship $F_{A}$ and $F_{B}$. The responses $R_{A}$ and $R_{B}$ are different even imposing the same challenge $C$ to them.

(4)Robustness. With the same challenge $x$ to PUF, the same response $y=\Gamma(x)$ is returned with a tolerance of a few errors. These errors should be within the range of distance measurement. Namely, the responses with subtle difference are close in distance measurement. The robustness of PUF is measured by intra hamming distance histogram. This feature is essential difference between PUF and pseudo-random number generator (PRNG). Almost all of existing PUF implementations are robust.

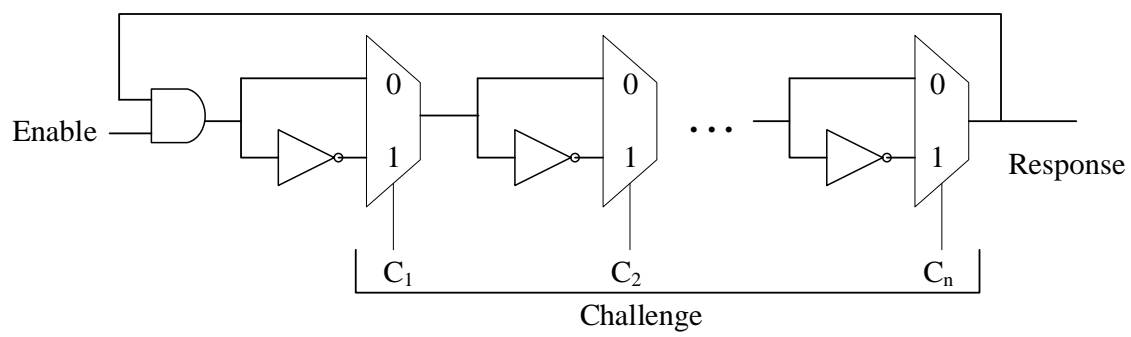

Fig.1. The CRP structure of PUF circuit

As shown in Fig.1, input and output signals of PUF circuit are respectively called Challenge and Response. The Challenge-Response Pair (CRP) exists when the circuit is power-on. PUF can be used to address the security issues in traditional cryptology, such as key storage. The PUF circuit of each electronic device has unique CRP, which is similar to biological identification information, such as fingerprint and voice. Consequently, one device can be distinguished from other devices according to CRP. Fig. 2 shows structure of PUF based identity authentication system. The CRPs of PUF circuit are stored in a public database. If copyright dispute occurs, the verifier can add some specific challenge information to PUF. The generated responses are then compared with the stored responses. If they are consistent, the identification is successful. Let's suppose PUF is used in television business. A user carries his identification card with PUF to bank. The card is then placed in a specific terminal with the database of PUF CRPs in manufacturing. According to the ID of this card, the terminal will impose a specific challenge to PUF. By comparing the returned response and the stored one, the correctness of this card can be verified. 
In this work, we summarize previous PUF based IP protection methods and analyze differences among various PUF techniques. Meanwhile, PUF techniques for real-time IP protection are studied. We predict that PUF based IP protection methods will provide good technical support for IP design and IC manufacture. Moreover, it is beneficial to sound development of future IC.

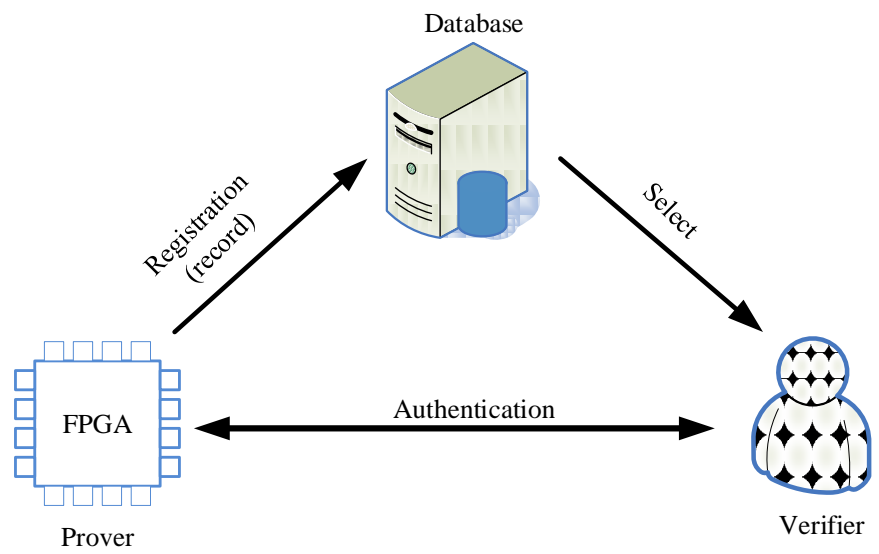

Fig.2. The structure of PUF based identity authentication system

\section{Concept and Classification}

\subsection{Concept of PUF}

PUF technique is a novel method to extract secret from complex physical system. It utilizes the random variation in manufacturing, intentionally added or carried by itself, to realize the uniqueness of a chip. The challenge-response pairs of PUF exist when the product is on power. So, the issue of key storage in IC can be addressed by PUF circuit when the product is running. The characteristic of no-cloning has greatly improved the security, making it superior in information security. 


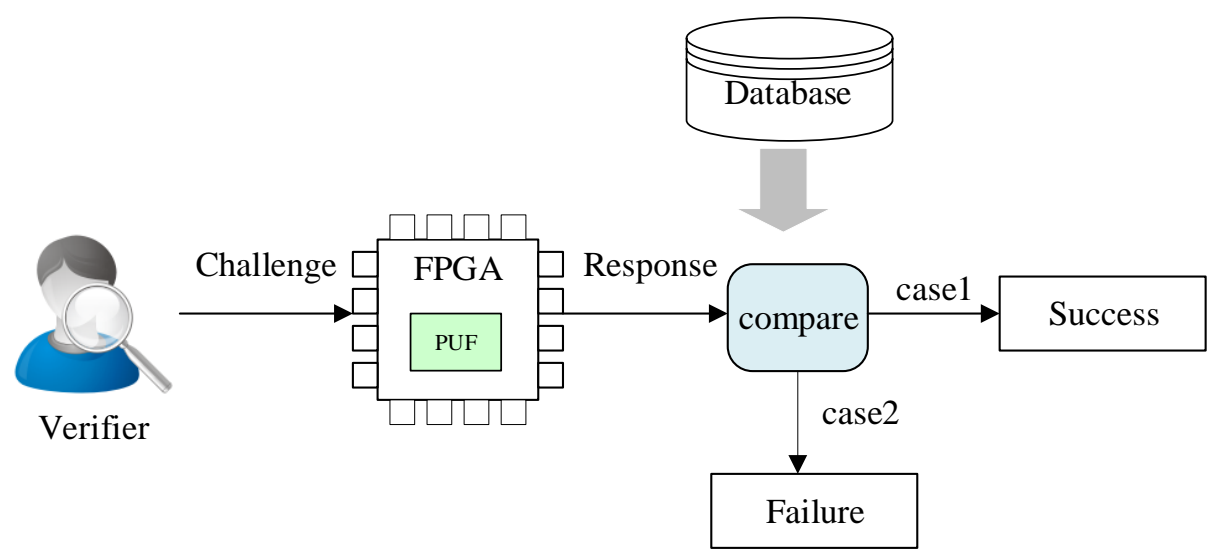

Fig.3. Identity authentication flow of PUF circuit

The identity authentication flow is shown in Fig.3. The challenge and response pairs of PUF in authentic device are recorded in database. When the device is used in an untrusted environment, PUF can provide an evidence in authentication. If the response is consistent with the stored one in database, authentication is successful (case1). Otherwise, it fails (case2). PUF utilizes manufacturing variations to authenticate device. In this way, it is unnecessary to store keys in memory. Meanwhile, the unclonable CRPs improve security of PUF circuit. It is predictable that the study on PUF-based IP protection will offer technical support to IP design and IC manufacturing. It plays an important role for healthy development of future IP circuit.

In recent years, many scholars have conducted researches on PUF based IP protection techniques. These techniques are classified into four categories based on features of PUF circuit.

\subsection{Classification of PUFs}

In this section, we have classified existing PUFs into four groups: Non-electronic PUF, physical characteristic based PUF, Memory based PUF and Delay based PUF. The detailed classifications are summarized in Fig.4. The related references are concretely illustrated in the subsections. 


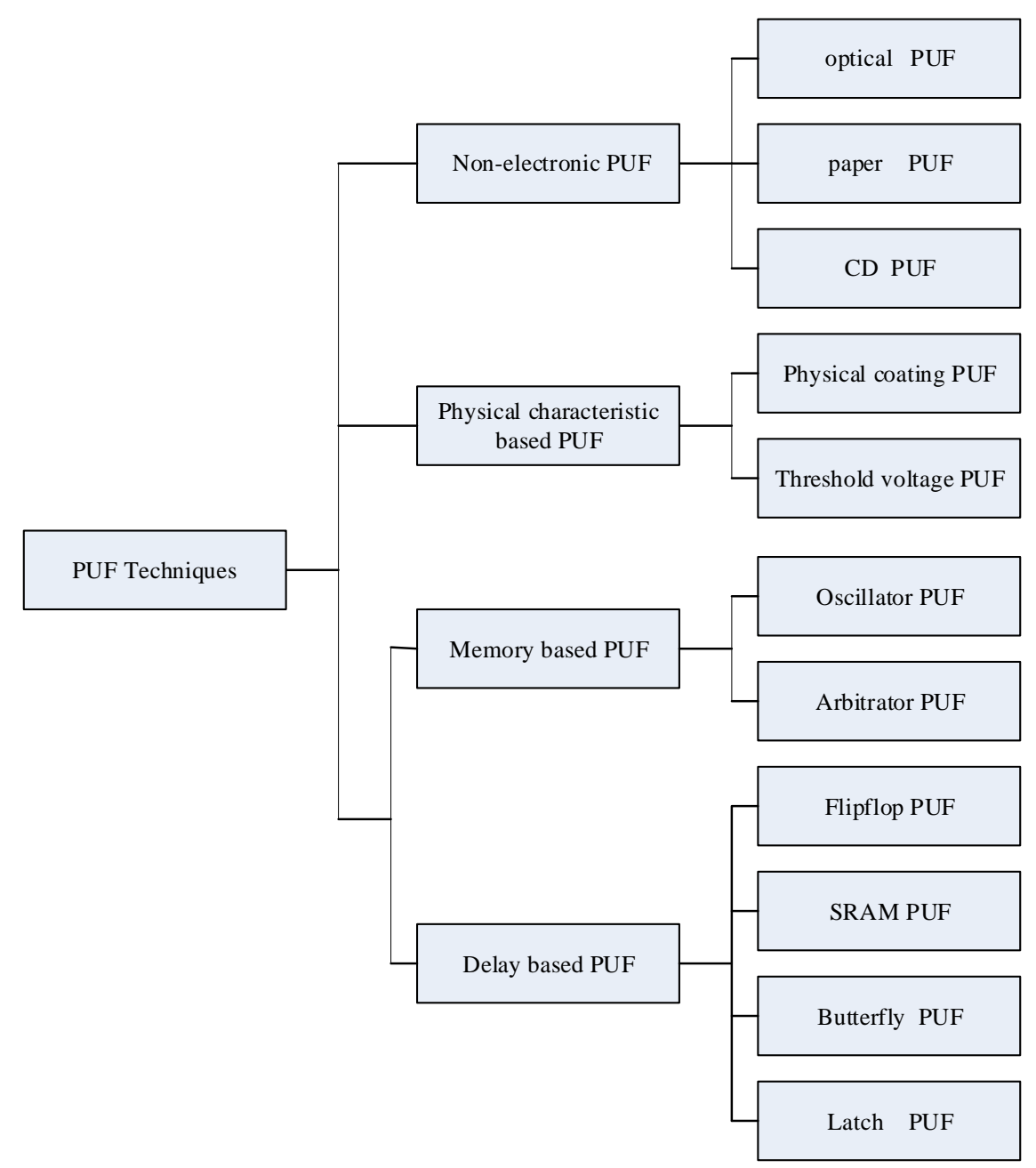

Fig.4. Architecture of PUF classification

\subsubsection{Non-electronic PUF}

A typical PUF technique is proposed by Pappu et al.[16] by utilizing optical physical characteristics. It is realized by an optical device with transparent material. There are optical scattering particles in transparent material, which can be randomly added to chip manufacturing technique. When a laser beam is cast over optical scattering particles, a few random speckles are produced. These speckles can record the position, angle or other parameters (such as amplitude and wave length) of the laser. 
They are utilized as secret keys and added into chip manufacturing. Since the response of optical PUF always depends on physical characteristic of optical token, the circuit may have two different CRPs. In this case, it is easy to prevent IP circuit from being copied by illegal users. Moreover, Lim et al. in reference [17] proposed a structure with randomly changing characteristics based on physical attributes of the token. The structure marks the characteristic for tamper-proofing in IP circuit by altering CRP of PUF.

In addition, Bulens [18] proposed a concept of paper PUF structure that utilizes the irregular physical features in paper file to prevent ownership from being forged by illegal attackers. For better security, Hammouri et al. presented a PUF circuit based on Compact Disc (CD) manufacturing model [19]. In manufacturing CD, the variable physical factor may affect the precision and length of data stored in CD. By utilizing the feature, the researchers can embed ownership information such as fingerprint in CD manufacturing model. After that, Rishab et al. presented a novel PUF identification technique based on optical physical characteristic in [20] for real-time protection of IP circuit. The technique has designed a small optical clearing token by randomly adding light scattering speckles. If the token is illuminated by a laser, a complex image with shades, called speckle, is shown. The speckle can be extracted by using the Gabor filter and a PUF response with optical physical characteristic will be output. Therefore, the researchers can identify the ownership by extracting the speckle. Wu et al. at Chinese Academy of Science [21] built a PUF circuit founded on random path delay model by considering the randomness of CMOS technology. Meanwhile, a novel enhanced protection scheme based on silicon PUF circuit is realized by interconnecting lines globally. However, the scheme has drawbacks on information hiding.

\subsubsection{PUF Techniques based on Physical Characteristic}

Generally, coating based PUF technique is implemented by integrating PUF into physical coating of IC. Tuyls et al. proposed a typical protective method of coating based PUF [22]. The principle is to generate a type of random medium particles in physical coating. Since random capacitance exists in these particles, researchers can measure the changes in capacitance by using capacitive sensors on metal layer of IC. In this case, various responses of coating PUF will be output. Consequently, the 
researches on coating PUF usually use capacitive sensor as the challenge signal and the response is the random capacitance produced in physical coating. In order to find more physical characteristics of coatings, Lofstrom et al. realized a secure PUF based protection by simulating the change of threshold voltage of IP circuit in the physical coatings [23].

In [24], Helinski et al. introduced a PUF protection method based on the change of resistance in physical coating of IP circuit. Moreover, [25] also presented a method to insert ownership information into coating material of intelligence card. The scheme can effectively protect hardware of IP circuit. In addition, there are many medium particles with random sizes and shapes in coating material. By using this feature, Majzoobi et al. proposed a PUF protective method based on wireless metal sensor cross structure [26]. The method shows the observed value of physical characteristics of coatings in oscilloscope. Since PUF is physically unclonable, it is difficult to produce another PUF with the same feature in physical coatings. Therefore, it is hard for illegal users to crack the characteristic information with description of physical characteristic. Meanwhile, Kumar,S et al. proposed a PUF coating structure with good features on tamper-proofing[27], which can be used as RFID label or secret key. When the PUF has suffered tampering attacks, the response will obviously be changed. A PUF for device authentication and secret key generation is proposed in [28]. The PUF is designed by using the intrinsic delay of physical coating of wire and transistor in IC manufacture. It is more suitable for low-cost individual PUF authentication.

The researchers have designed physical characteristic parameter of various PUF to be the challenge signal. The filter will output the response signal of IP circuit. The IP circuit can be further protected by recording these physical characteristics. In this case, the protection will be highly random and unique. However, the physical characteristic attributes have complex interaction. IP circuits are characteristic-dependent. Consequently, these schemes can be further investigated in terms of design practicability and application flexibility.

\subsubsection{Memory-based PUF Techniques}

Memory based PUFs are mainly implemented by oscillator and arbitrator. The structure of this PUF simultaneously activates two symmetrical path at work. 
Different input signals produce different delay paths. The arbitrator is implemented by a D-flipflop. It is placed at the end of the path and used to compare the orders of signals at both paths. To enhance the security, researchers usually increase complexity of the PUF circuit by adding some feedforward structure. The ring oscillator based PUF is developed on basis of arbitrator based PUF. It overcomes the limitation of delay based PUF due to the characteristics of oscillator.

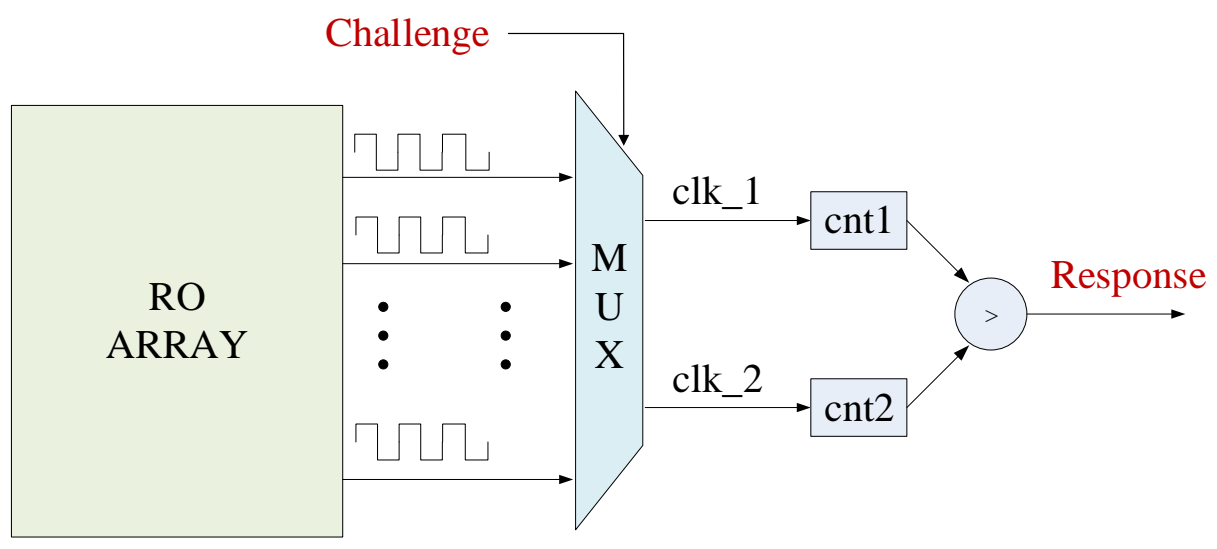

Fig.5. Architecture of oscillator based PUF

The oscillator based PUF is a technique that exploits the unique characteristic of physical frequency of single silicon chip in IC manufacturing. Fig.5 shows a PUF based on an array of $\mathrm{N}$ oscillators. These oscillators are identical to ensure the frequency differences are caused by manufacturing process. The high cost and stringent requirements for development environment limit the rapid application in secure chip protection. In order to provide enough CRPs for the design of oscillator frequency based PUF, researchers have improved the frequency generator. However, it leads to a sharp growth of overhead. In [29], Gassend et al. proposed a PUF by generating delay with oscillator. In chip manufacturing process, the controllability of oscillator is used to generate different delay time. It is unnecessary to operate on equipment for traditional chip manufacturing. The oscilloscope with high frequency is needed to observe the differences of chip delay, causing high power overhead. Suh and Qu in [30] and [31] proposed delay PUF schemes based on ring oscillator. The delay circuits of both schemes are designed by imitating arbiter PUF. Similarly, the improvements on security of both the schemes also cause an increase of resource overhead. 


\subsubsection{Delay-based PUF Techniques}

Gassend et al. [32] presented a safe and reliable "self-oscillation" method, which generated random bitstring with delay loop. It has good resistance against timing analysis attacks. In order to reduce power consumption, it is a trend for the development of IP circuit to design a PUF protection method based on FPGA. Guajardo et al. [33] improved SRAM based PUF to a Butterfly PUF (BPUF). The unstable cross coupling circuit is utilized in the improved PUF. In other words, the inverter is replaced by latch or flip-flop. The latch is employed to store circuit information, which can be cleared (the output is " 0 ") or reset (the output is " 1 "). In this way, the measurement can be realized without power-on. By comparing with self-oscillation PUF, BPUF is suitable for FPGAs of most categories. It is a new PUF structure for the protection of FPGA based IP core. Meanwhile, a practical PUF structure is proposed in [34], which can operate by binding the software to specific FPGA. When the ownership in a circuit is damaged after suffering illegal removal attacks, the ownership authentication of the circuit will be affected. To address this issue, Rührmair et al. [35] proposed a secure PUF model with resistance against attacks. Although it greatly improves the application value of PUF on FPGA device, it is easy to be forged due to the uniqueness of oscillation frequency.

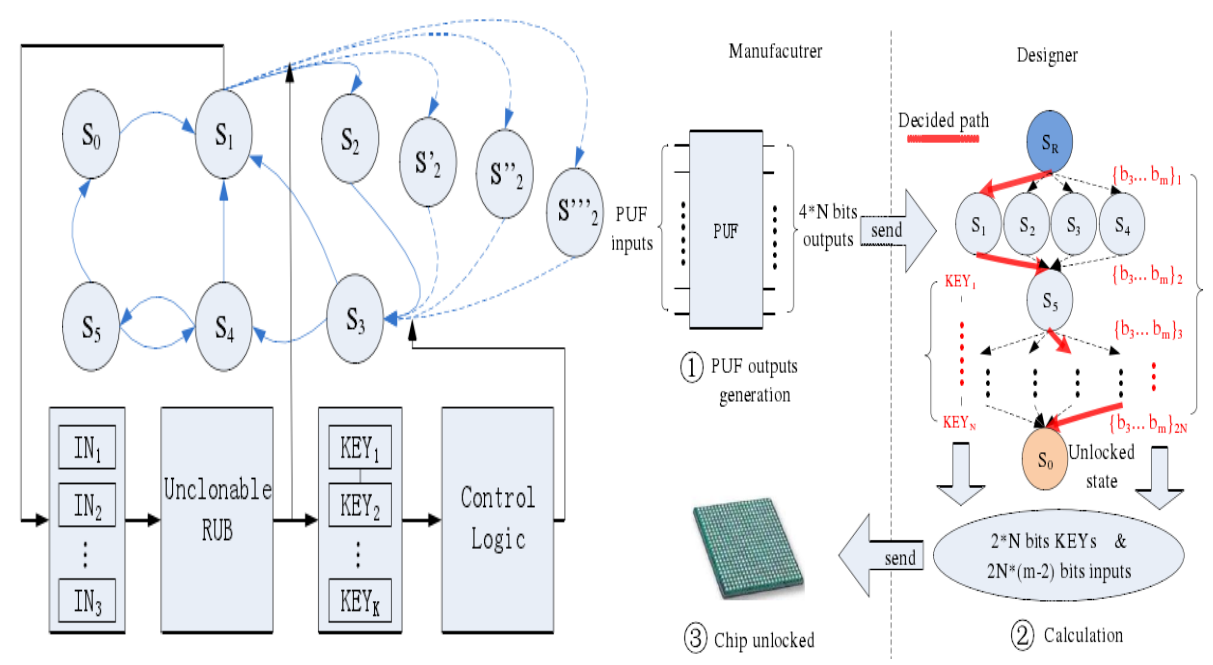


Fig.6. The unlock process based on n-layer PUF control structure against counterfeit ${ }^{[36]}$

Zhang et al. proposed a PUF scheme for IP core protection by using Finite State Machine (FSM) [36-39]. The FSM is added into original FSM as a lock. An FSM lock is generated by binding the added FSM to PUF. In this case, IP cores in each manufactured IC chip can be locked uniquely. The manufactured chip will jump to a fixed initial locking state when it is power on. By returning the unique PUF of IC to IP owner, the IC vendors could obtain a unique key for unlocking and the remaining inputs for transition. In this case, IC can be activated and unlocked. As shown in Fig.6, each chip needs to provide a specific collection of PUF inputs after being manufactured. When the chip is power on, each one is locked to a fixed initial state $S_{R}$. The chip can be activated by calculating the unlocking path. Consequently, the counterfeiting-proofing of chip will be realized.

Xiang et al. [40] proposed a multiple frequency slots based PUF by analyzing security issues of traditional oscillator based PUF. It is more difficult for the attacker to model this system. This architecture not only ensures the uniqueness, but also increases the safety. Li et al. [41] utilized the delay deviation caused by chip manufacturing as PUF production mechanism and improved arbiter based PUF by adding delay modules into path. The experiments on Field Programmable Gate Array (FPGA) have verified the security performance of improved scheme. Since delay-based PUF can be represented by linear inequality, it is utilized as a predictable function. Namely, a server can directly compute the predictable response of PUF without storing CRP. So, it makes PUF be light-weight. But it also brings some drawbacks. E.g., the adversary could easily simulate a predictable learning model by using the linear structure. By learning the CRPs of PUF, he can predict a new CRP to crack original secret key. Zhang et al [42] summarized the problems in existing PUFs and discussed the application prospect of PUF from the view of cryptology. Meanwhile, several meaningful research directions in this field are explored. The above methods have brought about low overhead and security of PUF circuit. However, the characteristic detection is relatively difficult, which imposes higher requirements and challenges to real-time detection and robustness of ownership information for PUF based protection method. 
Table 1. PUF implemented in the ASIC

\begin{tabular}{|c|c|c|c|c|c|}
\hline PUF class & PUF type & $\begin{array}{l}\text { Number of } \\
\text { instances } \\
\text { per ASIC }\end{array}$ & $\begin{array}{c}\text { Total } \\
\text { number } \\
\text { of } \\
\text { instances }\end{array}$ & $\begin{array}{l}\text { Challenge } \\
\text { space size }\end{array}$ & $\begin{array}{l}\text { Response } \\
\text { space size }\end{array}$ \\
\hline \multirow{2}{*}{$\begin{array}{c}\text { Delay-bas } \\
\text { ed }\end{array}$} & \multirow{2}{*}{$\begin{array}{c}\text { Arbiter } \\
\text { Ring } \\
\text { oscillator }\end{array}$} & 256 & 24.576 & $2^{64}$ & 2 \\
\hline & & 16 & 1.536 & $2^{15}$ & 2 \\
\hline \multirow{3}{*}{$\begin{array}{c}\text { Memory-b } \\
\text { ased }\end{array}$} & SRAM & $4(8 \mathrm{~KB})$ & 384 & $2^{11}$ & $2^{32}$ \\
\hline & Flip-flop & $4(1 \mathrm{~KB})$ & 384 & $2^{8}$ & $2^{32}$ \\
\hline & Latch & $4(1 \mathrm{~KB})$ & 384 & $2^{8}$ & $2^{32}$ \\
\hline
\end{tabular}

Table 1 shows two types of PUF circuit in Application Specific Integrated Circuit (ASIC), Delay-based and Memory-based, respectively. The results in the table compared the space between the interface of various PUF circuits and CRP for two PUF techniques. As shown, the Memory-based PUF has more complex structure than that of Delay-based PUF.

\section{PUF Security Analysis and Comparison}

\subsection{Analysis of Secure PUF Protocol}

The protocol design of PUF based identity authentication system generally is founded on random cipher. Assume that some sensitive information is known by user, such as cipher, PIN, the third party can affirm the ownership of PUF design by inquiring the information. If the ownership information about IP design is possessed by user, he can give some authentication rules. A real-time dynamic cipher will be obtained. In this case, real identity information about user can be also affirmed quickly. Current secure authentication of PUF circuits has good security. But there are also some limitations. For example, user's cipher may be easily attacked, revealed, lost or stolen. When user's identity information is sharing with others, the system cannot recognize whether the real user logs in the system. Meanwhile, the physical entities such as intelligent card in PUF system are easy to be lost or stolen. Once illegal attackers obtain the intelligent card and cipher, he can alter the user's cipher and log in remote authentication system. 
In applications such as authentication of crypto chip, the security realized by random dynamical cipher and intelligent card is not very high [43]. However, secure PUF circuit has lots of advantages, such as high recognition rate, reliable, practical, cannot be copied. Secure protection for chip can be realized by using the uniqueness of CRP of PUF. It is necessary to design a secure and reliable identity authentication protocol in some applications with requirement of high security.

In [44], the response characteristic information of user was extracted in registration and stored in characteristic library of the system. When a user logs in the third-party authentication system, the characteristic response of PUF circuit is extracted and compared with the stored one for authentication. If the similarity is greater than the threshold value, the identity authentication is successful. Otherwise, the identity authentication is failed.

\subsection{Analysis on Security and Functionality}

Any secure system with PUF requires an identification mechanism to verify legality. Under the situation of PUF based mobile authentication, we should ensure security of communication between low level hardware circuits as well as CRPs. Currently, many systems still utilize "encryption" for identity authentication. Users always set the same ID and password to log in different system. It brings convenience but poses a threat to information leaking. Also, users have different requirements for security level of data. Fig.7 shows a PUF based system for mobile identity authentication. A user can authenticate remote server with cell phone in real time. The design in Fig.6 is suitable for mobile identity authentication with different requirements. It will be an important application of PUF in field of security. 


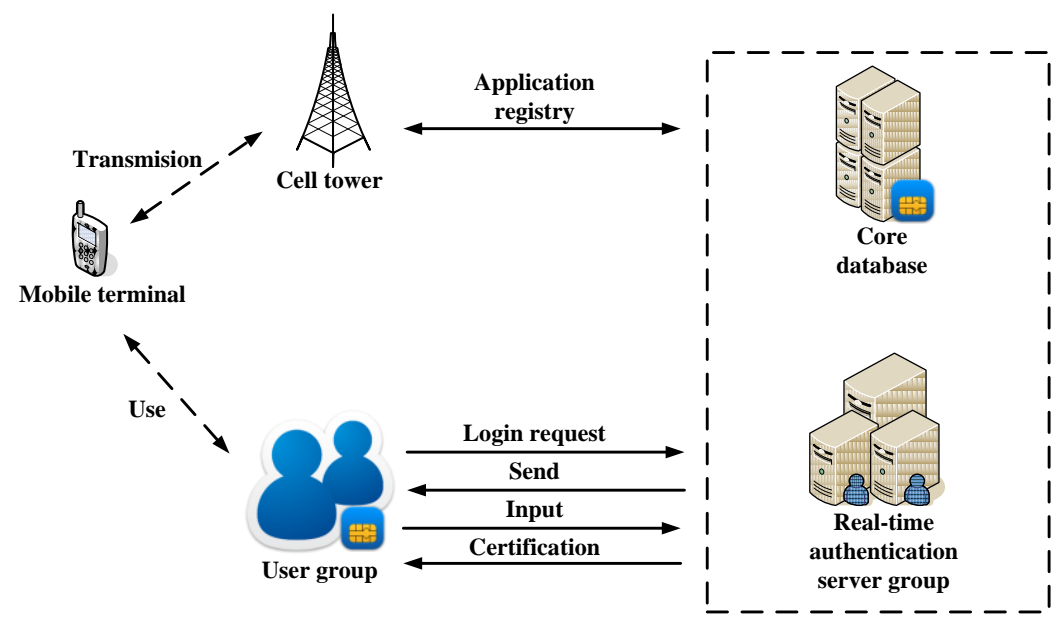

Fig.7. PUF based mobile authentication scheme

Several effective schemes for secure authentication are reported in $[43,44,45,46$, 47, 48, 49].The protocol in [43] has compared with that in [44] to evaluate secure performance and functional characteristics of PUF protocol. For analyzing computational complexity of PUF protocol, the time of one-way hash is denoted by Th. Sine XOR operation has low computational complexity, it can be ignored. In BEKC protocol, computations in registration, login and authentication are respectively denoted by $3 T h, 2 T h$ and $5 T h$. However, those are $4 T h, 4 T h$ and $7 T h$ in Kulseng's scheme. As known from the protocol design of Kulseng's scheme, the computational complexity is higher than that of BEKC protocol. Meanwhile, Fig.6 shows that the protocol can provide correct authentication and session key negotiation. It has good ability against man-in-middle attack. We make reference to scheme in $[48,49]$ and classify PUF based identity authentication system into three stages, registration, login and authentication respectively.

(1) Registration. A user $U_{i}$ selects an identifier $I D_{i}$ and secret key $P W_{i}$. Regenerating code $(R C)$ is used to calculate $A i=h(P I N \| R C)$. The sequence $(I D i, P W i, A i)$ is then sent to authentication center of IP copyright for registration. The center calculates $B i=h(I D i\|P W i\| A i), C i=h(I D i \| X S)$ and $D i=B i \oplus C i$. Here, $X S$ denotes the master key of authentication center. The center stores IDi into authentication server and sends $D i$ to user. The user will store $D i$ into chip. 
(2) Login. A user opens the login interface of authentication application in cell phone and enters $I D i$ and $P W i$. The application program extracts $R C$ and calculates $A i^{\prime}$ $=h(P I N \| C R), B i^{\prime}=h\left(I D i\|P W i\| A i^{\prime}\right), \quad M 1=D i \oplus B i^{\prime}$. A random number $r 1$ is also generated. $M 2$ is calculated by $M 2=M 1 \oplus r 1$. After that, $(I D i, M 2)$ is sent to authentication center to request login.

(3) Authentication. The authentication center checks if IDi is included in user list. If included, the center will generate a random number $r 2$ and challenge PUF to get response $\mathrm{Y}(r 2)$. Y $(r 2)$ is sent to user's cell phone. The user inputs number of response in application. Then, the application extracts random number $r 2^{\prime}$ and calculates $M 3=$ $h\left(M 1 \|\left(r 1 \oplus r 2^{\prime}\right)\right)$. With $M 3$, the authentication center can calculate $C i^{\prime}=h(I D i \| X S)$, $r 1^{\prime}=C i^{\prime} \oplus M 2$ and $M 3^{\prime}=h\left(C i^{\prime} \|\left(r 1^{\prime} \oplus r 2\right)\right) . M 3^{\prime}$ is compared to M3. If consistent, authentication center successfully verifies chip identity for user $U i$. Ui can activate authentication code at low level of chip and access the application system for authentication.

\subsection{Security Evaluation and Performance Analysis}

RFID protocol in [48] have assumed two keys in system, respectively the master key $X_{S}$ and key $y$. Here, $X_{S}$ is shared by registration center $R$ and sever $S_{i}$. $y$ is hold by registration center and stored in user's intelligent card $C_{i}$. Attackers cannot obtain $X_{S}$ from message $\left(I D_{i}, M 2, M 4, M 5\right)$ and $(M 10, M 11)$ since they are protected by one-way hash function.

(1) Security of session key. Previous security issues of session key mainly discuss the probability that the leakage of key value will not expose other session keys. In many protocols, the session key is protected by encryption techniques, such as hash [49]. If illegal attackers get some previous session keys, they will calculate key value by using techniques such as information reconfiguration. It brings great threat to the system on chip. Authors in [49] give a solution to this issue. The secret PUF response (key) can be ephemeral and immediately cleared after use. Therefore, it eliminates the security vulnerabilities of the permanent key management and exchange.

(2) Ability against counterfeit. If an illegal attacker stoles a chip with PUF, he will extract sensitive information in this chip and forge a new one. As we know, the response of PUF is unique. If an illegal user attacks the master key, he will not obtain 
the same response as the original chip. So, it is no use to interfere in chip authentication by changing initial key. Besides, due to the randomness of CRP of PUF based system, secure protocols in [50] have good ability against inner attacks even verification center can get some real key information of the users.

(3)Ability against Replay attacks. Attackers may pretend to be legal user and log in authentication server of system by replaying the previous intercepted login request of legal user. Generally, random numbers in authentication protocols are independently generated by user and server. These numbers are different with each other. So, the login request and authentication information transmitted in unsafe channel are various at each time. The attackers cannot replay the previous used information successfully. In protocol of [50], binding and locking mechanisms are proposed in order to prevent the replay attacks. It makes the reconfigurable PUFs generate significantly distinct responses.

We also compare performance of some authentication schemes [26, 33, 44,56 and 60]. The results are shown in Table.2. We compare these schemes in terms of timing, resource overhead, hardness of implementation, resistance to forgery and replay attacks. The overhead on timing and resource are inevitable in order to realize these schemes since the insertion of PUF circuit. But whether the overhead is high or low mainly depends on scheme itself. Moreover, the resistance against forgery of these schemes seem to be good owning to the features of PUF. But, the case of resistance against replay attacks is not so encouraging. Majority of schemes have low ability against this attacks except that in Zhang's scheme.

Table 2. Performance summary of several protocols

\begin{tabular}{|l|c|c|c|c|c|}
\hline performance & $\begin{array}{c}\text { Timing } \\
\text { overhead }\end{array}$ & $\begin{array}{c}\text { Resource } \\
\text { overhead }\end{array}$ & $\begin{array}{c}\text { hardness of } \\
\text { implementation }\end{array}$ & $\begin{array}{c}\text { Resistance } \\
\text { to forgery }\end{array}$ & $\begin{array}{c}\text { Resistance } \\
\text { to replay }\end{array}$ \\
\hline Guajardo 2007 & medium & high & Medium & High & low \\
\hline Zhang 2015 & low & low & hard & medium & high \\
\hline Majzoobi 2009 & Medium & Medium & Hard & high & low \\
\hline Safkhani 2011 & high & low & medium & medium & low \\
\hline Maes 2012 & medium & high & hard & high & medium \\
\hline
\end{tabular}

\section{Applications and Issues of PUF Techniques}

The applications and challenges of PUF techniques will be introduced in this section. 
The function of PUF in these applications and issues will be also discussed in details.

\subsection{Applications}

\subsubsection{Identity Authentication}

Identity authentication is the basic application of PUF. It is a useful anti-counterfeiting technology due to its no-cloning, anti-counterfeiting and light weight. At the registration stage, some CRPs of each PUF and identity of physical system will be stored in database [51-52]. At the authentication stage, a verifier selects a random CRP from the database and uses it to challenge the PUF of current system. If the observed response is close to the stored one in database, the authentication is successful. Otherwise, it fails. To resist replay attacks, each CRP of each PUF can only be used once and be deleted from database after verification. The false authentication may exist in this procedure. It includes false acceptance and false rejection. The former means that a false PUF is accepted in authentication. The latter rejects a true PUF in authentication. A threshold of true/false authentication depends on intra and inter hamming distance histograms. If both the histograms are not overlapped, a threshold can be selected in the difference of the histograms for correct authentication. If they are overlapped, the threshold should make a compromise between false acceptance and false rejection. In order to reduce the false acceptance and false rejection, the optimal selection is to set the threshold in the intersection of both the histograms. But the compromise depends on concrete application.

Owning to its uniqueness, PUF technique is prevalent in information security. PUF, used as an authentication module of identification card, is the simplest application. Recently, each identification card configures a RFID label. However, since the identification cards lack of hardware protection, the attackers may obtain the sensitive information carried by RFID label and clone a new card with the same function without notifying the card owner[53].

\subsubsection{Secure Transmission in Wireless Sensor Network}

The application of PUF in secure field of wireless sensor network has two advantages. (1) The anti-counterfeiting attribute of PUF can be used to address the 
issue on key storage. Any illegal attackers are required to match the CRPs. (2) In secure network transmission, attackers cannot probe secret key in communication due to the random characteristics of PUF. In addition, PUF-based authentication requires to update and delete secret key. So, it is unnecessary to permanently store the keys in storage of network. When the authentication starts, the PUF circuit can activate parts of keys, which effectively saves resources of network communication. eg. Liu, W describe a version incorporating Physically Unclonable Functions, able to resist simulation attacks in the event of an adversary being able to breach a trusted device to the extent of extracting embedded secret keys[54].

\subsection{Issues}

It is easy to forge a RFID label by using existing techniques, but hard to forge a PUF structure [55]. Therefore, the security is improved. However, in conclusion, the issues of existing PUF based IP protection methods still involve three aspects.

(1) Poor security and reliability. PUF as a novel technique for protecting ownership of IC chip attracts much attention in recent years. The security and reliability are important indicators to evaluate the maturity of PUF. However, existing PUF based IP protection methods provides few CRPs since the physical characteristic in response is singular. Consequently, illegal users may easily forge the characteristic by establishing mathematical model. From this view, more secure mechanisms should be integrated into PUF techniques for secure protection of PUF based IP circuit.

(2) High power overhead. At present, PUF based IP protection methods are almost realized by the change of signal propagation generated by oscillator. The feature of signal propagation change is actually the mapping function when the signal meets the electronic parameter of components at propagation path. The performance of a part of physical circuit will change when these parameters are suffered different influence, such as manufacturing discrepancy. In this case, the power overhead will be greatly increased, MASOUD ROSTAM proposes novel robust and low-overhead physical unclonable function (PUF)authentication and key exchange protocols that are resilient against reverse-engineering attacks, The protocols are executed between a party with access to a physical PUF (prover) and a trusted party who has access to the PUF compact model (verifier) [56].

(3) Limited practicability of detection. Generally, existing PUF techniques are only 
suitable for IP manufacturer to protect ownership. Legal IP user cannot authenticate and detect the ownership of the IP cores they bought in real time. It is unable for existing PUF protection methods to publicly detect ownership information in real time. Therefore, this technique is still not practical enough to be applied on a large scale

The existing PUF based IP protection methods still cannot meet the requirements of providing ownership protection for IC companies in terms of security, cost and practicability. Many issues with both theoretical complexity and practicality are required to be addressed, which bring great challenges to the completeness, confidentiality and application of IP protection methods.

\section{Discussion on secure PUF Techniques and Prospect}

IP protection technology is a new emerged field and attracts much attention in academic [57-58]. The complexity of IC design and the diversity of information processing make the PUF based IP protection more complex, but promising. Consequently, PUF based IP protection deserves further study. For PUF techniques, secure protection of signature hiding in integrated circuit should be first studied. Moreover, we should deeply analyze physical characteristics of PUF circuit and corresponding detection method.

Generally, physical characteristic library of original circuit is firstly established. Then, physical characteristic model can be built from the library. By using the characteristic classifier, the physical characteristic of original circuit is classified. Founded on this, techniques such as physical characteristic matching, reconfiguration, reversible equivalent replacement and content restoration are employed to design the models of signature hiding and detection based on physical characteristics. Consequently, the physical characteristic library is the foundation for theoretical research on PUF. The key point is to establish hiding model based on physical characteristics. Finally, the selection of a secure embedder and a real-time detector and the performance optimization are necessary to design and implement a successful system.

At initial stage of study on IP watermarking techniques in IC design, a large circuit characteristic library needs to be established. It can provide good foundation for physical characteristic library of the system. Moreover, the characteristic library chooses MCNC'91 benchmarks for testing. Berkeley SIS is employed to synthesize 
the circuit characteristic library. The formatted file in the library can be read and processed by using SIS. With the developments of PUF circuit, more circuits will be added in physical characteristic library. Four key techniques will be demonstrated as follows. The physical characteristic matching techniques of PUF circuits in MCNC'91 will be first introduced. The configuration techniques of eigenvector with multiple frequency slots, the equivalent replacement techniques based on reversible logical circuit and public verifiable real-time detection techniques are illustrated as well. The technological approaches are stated as follows.

1) PUF-based IP protection method based on delay characteristic matching model.

The PUF for IP protection may destroy the distribution law of non-periodic physical characteristics in original circuit, such as delay and noisy. Consequently, IP protection method based on non-periodic delay characteristic matching model can be designed to address this issue. The steps are stated as follows. Firstly, the non-periodic delay characteristic of original circuit is classified and the distribution law will be further researched. The delay characteristic matching models for various circuits are established. Founded on this, the attribution identification method based on the optimal delay model is proposed for PUF circuit IP protection. In addition, we compute the similarity of circuit characteristics in circuit library. A universal expression matching method for delay characteristic is deeply researched as well. Finally, we will provide a reliable robust PUF based scheme.

2) PUF-based IP protection method based on configurable characteristic of multiple frequency slots

The frequency characteristic generated by oscillator in PUF is very single. To address this issue, we have designed a new configurable oscillator. The little differences of frequencies between oscillators are investigated in order to establish a mathematical model of PUF circuit based on reconfigurable characteristic of multiple frequency slots. There are some inevitable manufacturing differences in each circuit under various frequencies. We can make statistics of these differences and research a random identification method of Challenge and Response Pair between different frequency bands of PUF circuit. In this case, the random value of input-output response will become larger, making it more complex for attackers. Since the functionality of PUF circuit is complex, we can make statistics of multiple frequency slots of PUF circuit and complete the reconfiguration calculation. At the same time, the corresponding model will be built in order to realize a signature hiding method with low-cost and high security based on reconfigurable characteristics of multiple 
frequency slots. At last, we further study the probability of circuit being attacked and complexity of secure PUF circuit model.

3) PUF-based IP protection method based on reversible circuit replacement.

To address the issues on high overhead of complex circuit and difficulty in function verification, some simple reversible combinational logic circuits can be used to replace complex sequential logic circuits in IP protection scheme. The dimensionality reduction method for characteristic vectors of circuit registers is also researched. To ensure correctness and reliability after inserting signature, we have designed an error correction detection mechanism for circuits. Since complex reversible logic circuits have various structures and functionalities, we should realize coding optimization in state space of characteristic vector in practical circuits. Furthermore, the mapping relationship of FSM (Finite State Machine) storage elements between complex sequential circuit and simple combinational circuit should be concentrated as well. Meanwhile, equivalent reversible replacement of registers and power optimization methods for complex sequential circuits are studied. Moreover, heuristic method is employed for researching characteristic vector of complex circuit. The equivalent replacement is transformed into state characteristic satisfiability problem of sub-circuits in complex circuit. In this case, the state characteristic of sub-circuit can be resolved by reversible resolver. The searching space of circuit state is simplified, achieving the goal of secure signature hiding in PUF circuit.

4) Publicly verifiable PUF based real-time detection for IP ownership

Illegal user may destroy signature in PUF circuit when he cannot obtain complete information. To address this issue, we propose a public real-time detection scheme for PUF circuit. Existing PUF techniques usually identify circuit ownership through the third party authentication institute. The credibility and controllability may bring new challenge to security of PUF detection system. To verify validity of ownership, a characteristic code of PUF circuit is generated randomly for public verification when designing the IC chip. When the chip is power-on, each circuit block is locked to a fixed state SR. The designer and manufacturer can publicly verify the ownership according to detection steps. Firstly, the designer will obtain the unique PUF characteristic response after the chip is power-on. The response is then regarded as evidence in zero-knowledge proof protocol. The designer's identity and secret key for detection are public in zero-knowledge protocol. The manufacturer will successfully obtain $\mathrm{N}$ bit physical characteristic response of PUF circuit and feedback to the designer. After that, effective state path information of FSM in PUF circuit will be 
computed with the response. Meanwhile, $\mathrm{N}$ bit secret key and $\mathrm{N}$-bit input value in path are returned to manufacturer of IC chip. After receiving the returned information, the manufacturer can activate verification mode. Therefore, the designer can publicly detect legal ownership of chip in real time.

5) Optimization on PUF characteristic model and Performance evaluation

Study on PUF characteristic model mainly concentrates on optimizations on kernel function and characteristic function of Support Vector Machine (SVM) classifier. Two aspects are included. (1)The goal is to improve the speed of characteristic classification and running of selection algorithm. Fatherly, redundancies and dependencies in characteristic information should be also identified. Firstly, we have learned standard online training algorithm of SVM classifier. Based on this, a dynamical characteristic selection algorithm is proposed to select characteristic information with high correlation, inner dependencies and redundancies. In addition, optimization method for function cache and deletion of redundant samples are researched to achieve optimal controlling parameters. (2) Optimization on hiding model for characteristic function in PUF circuit will be further studied. The characteristic controlling model of PUF circuit will also optimized. For better security, we also research optimization algorithm by employing SVM in order to make it superior in SVM training speed and accuracy of data collection.

The performance evaluation includes metrics in three aspects, stated as follows. Metric in security and efficiency is used to evaluate ability against attacks and metric in public detection is to evaluate real-time performance in detection. Moreover, we need to evaluate test the performances in terms of completeness, power overhead and transparency in public environment. With these metrics, a novel robust protection method and real-time detection system based on PUF circuit are established on FPGA platform.

\section{Conclusions}

The PUF-based secure hardware protection is an important technique in field of information security. IP owner can effectively protect IC ownership after manufacturing. Meanwhile, secure control in chip manufacturing can also be realized by utilizing a series of protection mechanisms, protocols and methods. In this work, the features of PUF in large scale IC are considered. We summarize this work in four 
parts. Firstly, we have classified and compared related references about PUF; secondly, illegal attacks in PUF based identity authentication protocol are introduced. The security of PUF circuit is analyzed as well. Thirdly, we have considered theories in IC design and information hiding, and the features of PUF. On this basis, current applications and issues of PUF technique were summarized and concluded. Finally, we envision the future of PUF techniques. Although much work has been done in this field, there are lots of issues to be addressed. The review in this work can provide guidance for PUF based ownership protection in semiconductor field.

\section{Acknowledgements}

This work is supported by the National Science Foundation of China (No.61572188), the Scientific Research Fund of Hunan Provincial Education Department (Grant14A047), the China Postdoctoral Science Foundation funded project (Grant140778) and Scientific Research Fund of Hunan Provincial Education Department (Grant10C0689).

\section{References}

1. Zhang, L. and Chang, C. H. A Pragmatic Per-Device Licensing Scheme for Hardware IP Cores on SRAM-Based FPGAs. IEEE TRANSACTIONS ON INFORMATION FORENSICS AND SECURITY, VOL. 9, NO. 11, pp:1893-1905.(2014).

2. Katzenbeisser, S., Kocabas, U., et al. Recyclable PUFs: Logically reconfigurable PUFs. J. Cryptogr. Eng., 2011, Vol. 1, No. 3, 177-186.

3. Kahng, A. B., Lach, J., Mangione-Smith, W. H., et al.: Constraint-based Watermarking Techniques for Design IP Protection. IEEE Transactions on Computer-Aided Design of Integrated Circuits and Systems, Vol. 20, No.10, 1236-1252. (2001).

4. Abdel-Hamid, A. T., Tahar, S., and Aboulhamid, E.M.: A Survey on IP Watermarking Techniques. International Journal on Design Automation for Embedded Systems, Springer-Verlag press, Vol. 9, No. 3, 211-227. (2005).

5. Li, D., Zheng, W., Zhang, M.: Advancement on IP Watermarking Techniques. Journal of Circuits and Systems, Vol.12, No.4, 84-92. (2007).

6. Long, J., Zhang, D., Liang, W., Li, W. A Fault-Tolerant Watermarking Algorithm for FPGA IP Protection. 2015 IEEE TrustCom, 230-237. (2015). 
7. Xu, J., Long, J., Peng, L.: A High-Capability Scattered IP Watermarking Algorithm in FPGA Design. Journal of Computer Research and Development, Vol.50, No. 11, 2389-2396. (2013).

8. Saha, D., Sur Kolay S.: Secure Public Verification of IP Marks in FPGA Design through a Zero-knowledge Protocol. IEEE T VLSI Syst, Vol. 20, 1749-1757. (2012).

9. Cui, A., Chang, C. H., Tahar, S.: A Robust FSM Watermarking Scheme for IP Protection of Sequential Circuit Design. IEEE Transactions on Computer-Aided Design of Integrated Circuits and Systems, Vol. 30, No. 5, 678-690. (2011).

10. Liang, W., Sun, X., Xia, Z., Sun, D., Long, J.: A Chaotic IP Watermarking in Physical Layout Level Based on FPGA. Radioengineering, Vol. 20, No. 1, 118-125. (2011).

11. Wei, L., Dafang, Z., zhiqiang, Y., Wenwei, L.: A IP Watermarking Scheme Based on Self-recovery of Secret Information. Journal of Computational and Theoretical Nanoscience, Vol.11 No.8, 1127-1731. (2014).

12. J. Guajardo, S. S. Kumar, G.-J. Schrijen, and P. Tuyls, "FPGA intrinsic PUFs and their use for IP protection," in Proc. Workshop Cryptograph. Hardw. Embedded Syst., Vienna, Austria, pp. 63-80. (2007).

13. S. Pappala, M. Niamat, and W. Sun, "FPGA based key generation technique for anti-counterfeiting methods using physically unclonable functions and artificial intelligence," in Proc. Int. Conf. Field- Program. Logic Appl., Oslo, Norway, pp. 388-393. (2012).

14. Maiti A, Kim I, Schaumont P. A robust physical unclonable function with enhanced challenge-response set. IEEE Trans.Inf. Forensics Secur., 7(1): 333-345. (2012).

15. Maiti A, Schaumont P. Improved ring oscillator PUF: An FPGA-friendly secure primitive. Journal of Cryptology, 2011,24(2): 375-397.

16. Pappu, R. S. , Recht, B. , Taylor, J., Gershenfeld, N. Physical one-way functions . Science 297 (5589), 2026-2030. (2002).

17. Lim, D., Lee, J. W., Lim, D., Gassend, b., Suh, G. E., van Dijk, M., Devadas, S. Extracting secret keys from integrated circuits. IEEE Transactions on Very Large Scale Integration(VLSI)Systems, Vol.13, No. 10, 1200-1205. (2005).

18. Bulens, P., Standaert, F. X., Quisquater, J.: How to Strongly Link Data and Its Medium: the paper case. IET Information Security, Vol.4, No. 3, 125-136. (2010).

19. Hammouri, G., Dana, A., Sunar, B.: CDs have fingerprints too.Proceedings of the 11th International Workshop on Cryptographic Hardware and Embedded Systems. Berlin: Springer, 348 -362. (2009). 
20. Rishab, N. John, S.: A Theoretical Analysis: Physical Unclonable Functions and The Software Protection Problem, IEEE Symposium on Security and Privacy Workshops, 1-11. (2012).

21. Wu, Z., Duan, C.: Design of Silicon Physical Unclonable Functions using Global Interconnect Stochastic Model. Microelectronics and Computer, Vol. 29, No. 12, 36-42. (2012).

22. Tuyls, P., Schrijen, G. J., Koric, B., et al.: Read-proof hardware from protective coatings. Cryptographic Hardware and Embedded Systems Workshop. New York: ACM, 369 -383. (2006).

23. Lofstrom, K., Daasch, W. R., Taylor, D.: IC identification circuit using device mismatch . Proceedings of Solid-State Circuits Conference. Washington, DC: IEEE Computer Society, 372 -373. (2000).

24. Helinski, R., Acharyya, D., Plusquellic, J.: A physical unclonable function defined using power distribution system equivalent resistance variation.Proceedings of the 46th Annual Design Automation Conference. New York: ACM, 676-681. (2009).

25. Majzoobi, M., Koushanfar, F., Devadas S.: FPGA PUF using programmable delay lines. In : Information Forensics and Security(WIFS), 1-6. (2010).

26. Majzoobi, M., Koushanfar, F., Potkonjak, M. Techniques for design and implementation of secure reconfigurable PUFS. ACM Transactions on Reconfigurable Technology System, Vol.2, No.1, 1 -13. (2009).

27. Kumar, S., Guajardo, J., Maes, R., Schrijen, GJ., Tuyls, P.: The butterfly puf: Protecting ip on every FPGA, In: HOST, 67-70. (2008).

28. Edward Suh, G., Srinivas D.: Physical Unclonable Functions for Device Authentication and Secret Key Generation, Proceedings of the 44th annual Design Automation Conference, 9-14. (2007).

29. Gassend, B.: Physical random functions. Cambridge,USA: Massachusetts: Massachusetts Institute of Technology, 97-112. (2003).

30. Suh, GE., Devadas, S.: Physical Unclonable functions for device authentication and secret key generation.Design Automation Conference. New York: ACM, 9 -14. (2007).

31. Qu, G., and Yin, C.E.: Temperature-aware cooperative ring oscillator PUF. In Hardware-Oriented Security and Trust, HOST'09. IEEE International Workshop. (2009).

32. Gassend, B., Clarke, D., Vandijk, M., et al.: Delay-based circuit authentication and applications.ACM Symposium on Applied Computing. New York: ACM, 294-301. (2003). 
33. Guajardo, J., Kumar, S. S., Schrijen, G. J, Tuyls, P. FPGA intrinsic PUF and their use for IP protection. In: Paillier, P. , Verbauwhede, I. CHES2007. LNCS, vol. 4727, 63-80. (2007).

34. Rostami, M. Majzoobi, M. ; Koushanfar, F. ; Wallach, D.S. ; Devadas, S. Robust and Reverse-Engineering Resilient PUF Authentication and Key-Exchange by Substring Matching. IEEE Transactions on Emerging Topics in Computing, Vol. 2, No. 1, 37-49. (2014).

35. Rührmair, U., Sölter, J., Sehnke, F., et al. PUF Modeling Attacks on Simulated and Silicon Data. IEEE Transactions on Information Forensics and Security, Vol. 8, No.11, 1876-1891. (2013).

36. Zhang, J., Qu, G. et al. A Survey on Silicon PUFs and Recent Advances in Ring Oscillator PUFs, Journal of Computer Science and Technology, Vol. 29, No.4, 664-678. (2014).

37. Wenjie Che, Yaping Lin, Aobo Pan, Jiliang Zhang. A Robust Hierarchical FSM Structure for Active IC Metering. Information Technology Journal, Vol.12, No.6, 1107-1115. (2013).

38. Zhang, J., Lin, Y., Lyu, Y., Qu, G., et al.: FPGA IP Protection by Binding Finite State Machine to Physical Unclonable Functions. In 23rd IEEE International Conference on Field Programmable Logic and Applications (FPL'13), Porto, Portugal, 1-4. (2013).

39. Zhang, J., Lin, Y., Lyu, Y., Qu, G., et al.: Binding Hardware IPs to Specific FPGA Device via Inter-twining the PUF Response with the FSM of Sequential Circuits. In 21st IEEE International Symposium on Field-Programmable Custom Computing Machines (FCCM'13), Seattle, USA, 227-231. (2013).

40. Xiang, Q., Zhang, P., Ouyang, D., et al.: Multiple Frequency Slots based Physical Unclonable Functions. Journal of Electronics and Information Technology, Vol. 34, No. 8, 2007-2012. (2012).

41. Li, R.: Improvement of Arbiter based PUF. China Sciencepaper. Vol. 6, No. 10, 707-711. (2011).

42. Zhang, Z., Guo, Y.: Survey of Physical Unclonable Function. Journal of Computer Applications, Vol.32, No. 11, 3115-3120. (2012).

43. Kulseng, L., Yu, Z., Wei, Y., and Guan, Y.: Lightweight Mutual Authentication and Ownership Transfer for RFID systems. In The proceedings of IEEE INFOCOM, 1-5. (2010).

44. Safkhani, M., Nasour B., and Majid N.: Security Analysis of a PUF based RFID Authentication Protocol. IACR Cryptology ePrint Archive2011. (2011). 
45. Zhang, J., Lin Y., Lyu, Y., Qu, G.. A PUF-FSM Binding Scheme for FPGA IP Protection and Pay-Per-Device Licensing. IEEE Transactions on Information Forensics and Security, v 10, n 6, p 1137-1150. (2015).

46. Mehrdad Majzoobi, Masoud Rostami, Farinaz Koushanfar, Dan S. Wallach, Srinivas Devadas. Slender PUF Protocol: A lightweight, robust, and secure authentication by substring matching. IEEE Symposium on Security and Privacy Workshops, 33-44. (2012).

47. Xiong Li, Yongping Xiong, Jian Ma, Wendong Wang. An efficient and security dynamic identity based authentication protocol for multi-server architecture using smart cards. Journal of Network and Computer Applications, Vol.35, No. 2, pp. 763-769. (2012).

48. Xiong Li, Jianwei Niu, Jian Ma, Wendong Wang, Chenglian Liu. Cryptanalysis and improvement of a biometrics-based remote user authentication scheme using smart cards. Journal of Network and Computer Applications, Vol.34, No.1, pp.73-79. (2011).

49. Xiong Li, Jianwei Niu, Saru Kumari, Muhammad Khurram Khan, Junguo Liao,Wei Liang. Design and analysis of a chaotic maps-based three-party authenticated key agreement protocol. Nonlinear Dynamics, Vol. 80, No. 3, pp. 1209-1220. (2015).

50. Xiong Li, Jianwei Niu, Junguo Liao, Wei Liang. Cryptanalysis of a dynamic identity based remote user authentication scheme with verifiable password update. International Journal of Communication Systems, Vol. 28, No. 2, pp. 374-382. (2015).

51. Xiong Li, Jianwei Niu, Saru Kumari, Junguo Liao, Wei Liang. An enhancement of a smart card authentication scheme for multi-server architecture. Wireless Personal Communications, Vol. 80, No. 1, pp. 175-192. (2015).

52. Xiong Li, Jianwei Niu, Yazhi Liu, Junguo Liao,Wei Liang. Robust dynamic ID-based remote user authentication scheme using smart cards. International Journal of Ad Hoc and Ubiquitous Computing, Vol. 17, No. 4, pp. 254-264. (2014).

53. Zhang, J., Lin, Y. Reconfigurable Binding against FPGA Replay Attacks. ACM Transaction on Design Automation of Electronic Systems, Vol. 20, No.2, pp.1-20. (2015).

54. Liu, W, Zhang, Z, Li, M, et al. A Trustworthy Key Generation Prototype Based on DDR3 PUF for Wireless Sensor Networks.Sensors,Vol:14, No.7, pp.11542-11556.(2014).

55. Xiong Li, Muhammad Khurram Khan, Saru Kumari, Junguo Liao, Wei Liang. Cryptanalysis of a Robust Smart Card Authentication Scheme for Multi-server Architecture. In International Symposium on Biometrics and Security Technologies (ISBAST 2014), pp. 120-123. (2014). 
56. Maes, R., Schellekens, D., Verbauwhede, I. A Pay-per-Use Licensing Scheme for Hardware IP cores in Recent SRAM-Based FPGAs. IEEE Transactions on Information Forensic and Security, vol.7, no.1, pp.98-108. (2012).

57. R. Pappu, B. Recht, J. Taylor, and N. Gershenfeld, "Physical one-way functions," Science, vol. 297, no. 5589, pp. 2026-2030.(2002).

58. H. Salmani, M. Tehranipoor, and J. Plusquellic, "A novel technique for improving hardware trojan detection and reducing trojan activation time," IEEE Trans. Very Large Scale Integr. Syst. vol. 20, no. 1, pp. 112-125. (2012). 